\title{
DYSELECTROLYTAEMIA IN STABLE AND ACUTE EXACERBATION CHRONIC OBSTRUCTIVE PULMONARY DISEASE PATIENTS- A CROSS SECTIONAL STUDY
}

\author{
Supriya Adiody1, Fabeena M. S2, Narmadha M. P3, Varghese P. $R^{4}$
}

1 Professor \& HOD, Department of Pulmonary Medicine, Jubilee Mission Medical College \& Research Institute, Thrissur, Kerala. 2M. Pharm Student, Department of Pharmacy Practice, Nehru College of Pharmacy, Pampady, Thrissur, Kerala. ${ }^{3}$ Professor \& HOD, Department of Pharmacy Practice, Nehru College of Pharmacy, Pampady, Thrissur, Kerala. ${ }_{4}^{4}$ Research Co-ordinator, Jubilee Centre for Medical Research, Jubilee Mission Medical College \& Research Institute, Thrissur, Kerala.

\begin{abstract}
\section{BACKGROUND}

The Global Initiative for Chronic Obstructive Lung Disease (GOLD) recently defined COPD as "A common preventable and treatable disease characterised by persistent airflow limitation that is usually progressive and associated with an enhanced chronic inflammatory response of the airways and the lung to noxious particles or gases." Though the COPD patients mostly present with the features of acute respiratory infections (productive cough, dyspnoea, etc.), there may be a number of metabolic derangements arising out of the disease process or as a consequence of the therapy instituted like dyselectrolytaemia.

This study is aimed at comparing the levels of Sodium, Potassium and Magnesium in Stable COPD and COPD Exacerbation (AECOPD) Patients.
\end{abstract}

\section{MATERIALS AND METHODS}

The study was an observational study of 100 COPD patients (50 stable COPD and 50 AECOPD patients) of Pulmonology Department in Jubilee Mission Medical College and Research Institute, Thrissur. Using a specially designed Proforma and patients details such as age, duration of disease, BMI, X-ray, levels of serum sodium, potassium, magnesium were recorded.

\section{RESULTS}

Out of 100 patients in the study, 90\% were male and remaining $10 \%$ were female. Common presenting symptoms were dyspnoea, muscle weakness, chest pain and cough and they were more severe in inpatients (AECOPD). Comorbid conditions such as hypertension, diabetes mellitus and coronary artery disease added to the insult leading to hospitalisation. Dyselectrolytaemia was more severe in acute exacerbated chronic obstructive pulmonary disease patients as compared to stable chronic obstructive pulmonary disease patients.

\section{CONCLUSION}

There were changes in levels of sodium, potassium and magnesium in AECOPD patients compared to stable COPD patients. Timely screening with earlier detection and intervention would be useful in controlling AECOPD.

\section{KEYWORDS}

Chronic Obstructive Pulmonary Disease (COPD), Acute Exacerbation COPD (AECOPD), Levels of Sodium, Potassium and Magnesium.

HOW TO CITE THIS ARTICLE: Adiody S, Fabeena MS, Narmadha MP, et al. Dyselectrolytaemia in stable and acute exacerbation chronic obstructive pulmonary disease patients- A cross sectional study. J. Evolution Med. Dent. Sci. 2017;6(28):2296-2299, DOI: $10.14260 /$ Jemds/2017/494

\section{BACKGROUND}

Chronic Obstructive Pulmonary Disease (COPD) is the sixth leading cause of death and therefore a disease of increasing public health importance around the world. Estimations of Global Initiative for Chronic Obstructive Lung Disease (GOLD) suggest that COPD will rise to the third most common cause of death worldwide by 2020 .

Financial or Other, Competing Interest: None

Submission 31-01-2017, Peer Review 15-03-2017,

Acceptance 22-03-2017, Published 06-04-2017.

Corresponding Author:

Dr. Supriya Adiody,

Professor \& HOD,

Department of Pulmonary Medicine,

Jubilee Mission Medical College \& Research Institute,

Thrissur, Kerala-680005,

India.

E-mail: supriyaadiody@yahoo.in

DOI: $10.14260 /$ jemds $/ 2017 / 494$

\section{(c) $(1) \ominus$}

Acute exacerbations that compromise quality of life, accelerate decline in respiratory functions, and increased economic costs may occur during the course of stable COPD. COPD exacerbation was defined as an acute worsening of respiratory symptoms (increased dyspnoea, increased cough or change in amount, and purulence of sputum) that was beyond normal day-to-day variations of symptoms. COPD acute exacerbation (COPD-AE) frequently appears with respiratory tract infections. It is a significant cause of mortality and morbidity.

The worldwide increase in COPD prevalence renders disease exacerbation an increasingly worrying phenomenon for clinicians, patients, healthcare organisations, and society in general. As a result, there is a mounting interest not only in designing optimal COPD treatment approaches but also in preventing its exacerbations. Magnesium $(\mathrm{Mg++})$ is an intracellular cation which is involved in the regulation of bronchial tone, mast cell secretion, neuromuscular activity and respiratory muscle function. 
Acute exacerbations occur commonly in patients with COPD. Exacerbations may cause specific signs and symptoms, such as increased dyspnoea, productive cough with altered sputum, and fever. Alternatively, the symptoms may be more nonspecific, such as malaise, fatigue, insomnia or sleepiness, and depression. Although such exacerbations are associated with impaired lung function, it is estimated that only $50 \%$ of all exacerbations are reported to physicians. Owing to the associated morbidity and mortality, health-care utilisation is usually significantly increased during an exacerbation. Hence, our study aims to establish whether dyselectrolytaemia and its correction have an impact in the management of COPD patients.

With this background, in the present study, we attempted to measure the concentration of major serum electrolytes (sodium, magnesium, potassium) in COPD patients with acute exacerbation and compare those findings with that of stable COPD to verify the occurrence of dyselectrolytaemia in the said group of patients which may be one of the major risk factors for increased mortality and morbidity in them.

\section{MATERIALS AND METHODS}

The study was carried out in the outpatients and inpatients of the Pulmonology Department at Jubilee Mission Medical College and Research Institute, Thrissur, Kerala. It was an observational study conducted in 100 COPD patients (50 Stable COPD \& 50 AECOPD patients) to compare the levels of Sodium, Potassium and Magnesium. Study has been carried out for a period of 5 months from February 2015 to June 2015.

Case Group subjects presented with an exacerbation of COPD requiring hospitalisation. Control group form the patients who presented to routine OPD for Followup. All those patients who gave informed consent were enrolled into the study. 100 patients were enrolled in the study and underwent assessment tests for Sodium, Potassium and Magnesium. Data such as age, gender, family history, duration of COPD Symptoms, body mass index were noted and documented. Majority of patients were already detected COPD. Only few were newly detected. Sodium, Potassium and Magnesium levels were checked. The data was analysed using t-test with SPSS version 20.

\section{RESULTS}

In this study, 100 patients (50 COPD \& 50 AECOPD) were included. The mean age of outpatients was $62 \pm 8.17$ and inpatients was $64.74 \pm 8.68$. Higher number of patients were in the age group of $50-70$ years (76\%) in OP and $60-70$ years $(44 \%)$ in IP. In outpatients, $90 \%$ were male \& $10 \%$ were female. In inpatients, $88 \%$ were male $\& 12 \%$ were female. The BMI analysis showed that in outpatients, $86 \%$ were normal weight, $10 \%$ were overweight and $4 \%$ were obese. In inpatients, $96 \%$ were normal weight, $2 \%$ were overweight and $2 \%$ were obese. Smoking behaviour is given in Table 1.

\begin{tabular}{|c|c|c|c|}
\hline & Smokers & Past Smokers & Non-smokers \\
\hline Outpatient & 66 & 16 & 18 \\
\hline Inpatient & 68 & 12 & 20 \\
\hline Table 1. Frequency of Smoking Behaviour in Percentage \\
\hline
\end{tabular}

In outpatients, $28 \%$ had COPD symptoms for $<1$ year, $14 \%$ had for $1-2$ years, $28 \%$ had $2-5$ years and $30 \%$ had for $\geq$ 5 years. In inpatients, $28 \%$ had COPD symptoms for $<1$ year,
$8 \%$ had for $1-2$ years, $26 \%$ had for $2-5$ years and $38 \%$ had for $\geq 5$ years. COPD related symptoms presented by the study group is presented in table 2 . Chest pain was significantly higher in inpatients. P-value for other symptoms like cough, muscle weakness and dyspnoea showed no significance in the two groups.

\begin{tabular}{|c|c|c|c|c|c|}
\hline & \multicolumn{2}{|c|}{ Outpatient } & \multicolumn{2}{c|}{ Inpatient } & \multirow{2}{*}{ p value } \\
\cline { 2 - 5 } & Present & Absent & Present & Absent & \\
\hline Chest pain & 16 & 84 & 38 & 62 & $0.013^{*}$ \\
\hline Cough & 28 & 72 & 46 & 54 & 0.062 \\
\hline $\begin{array}{c}\text { Muscle } \\
\text { weakness }\end{array}$ & 28 & 72 & 36 & 64 & 0.391 \\
\hline Dyspnoea & 100 & - & 100 & - & - \\
\hline \multicolumn{5}{|c|}{$\begin{array}{c}\text { Table 2. Frequency of COPD } \\
\text { Related Symptoms in Percentage }\end{array}$} \\
\hline
\end{tabular}

* Significant at 0.05

This study shows that $36 \%$ patients were admitted in ICU and $64 \%$ were admitted in the ward. Among inpatients, 13 required Non-invasive ventilation (NIV). Of the patients who needed NIV care, $77 \%$ had a serum sodium level of $<136$ $\mathrm{mEq} / \mathrm{L}$ and the remaining patients $23 \%$ had normal Sodium levels. Among these patients, $69 \%$ had serum potassium levels of $<3 \mathrm{mEq} / \mathrm{L}$ and the remaining had normal levels. Magnesium levels were below $1.08 \mathrm{mEq} / \mathrm{L}$ in $62 \%$ of patients indicating that when there was associated dyselectrolytaemia, it led to muscle weakness, disorientation and possibly increased need of NIV. Similarly, dyselectrolytaemia was also associated with increased duration of hospital stay as indicated in table 3 .

\begin{tabular}{|c|c|c|c|c|c|}
\hline \multicolumn{2}{|c|}{ Serum level } & \multicolumn{2}{|c|}{ Hospital Stay } & \multirow{2}{*}{ Total } & \multirow{2}{*}{ p-value } \\
\cline { 2 - 5 } & $<136$ & 0 & 36 & 36 & \\
\hline \multirow{2}{*}{$\begin{array}{c}\text { S. Sodium } \\
(\mathrm{mEq} / \mathrm{L})\end{array}$} & $136-148$ & 1 & 13 & 14 & \multirow{2}{*}{0.105} \\
\cline { 2 - 5 } & $>148$ & 0 & 0 & 0 & \\
\hline \multirow{2}{*}{$\begin{array}{c}\text { S. Potassium } \\
(\mathrm{mEq} / \mathrm{L})\end{array}$} & $<3.6$ & 0 & 30 & 30 & \multirow{2}{*}{0.216} \\
\cline { 2 - 5 } & $3.6-5$ & 1 & 19 & 20 & \\
\cline { 2 - 5 } & $>5$ & 0 & 0 & 0 & \\
\hline \multirow{2}{*}{$\begin{array}{c}\text { S. Magnesium } \\
(\mathrm{mEq} / \mathrm{L})\end{array}$} & $<1.8$ & 0 & 23 & 23 & \multirow{3}{*}{0.624} \\
\cline { 2 - 5 } & $1.8-2.6$ & 1 & 25 & 26 & \\
\cline { 2 - 5 } & $\geq 2.6$ & 0 & 1 & 1 & \\
\hline \multicolumn{3}{|c|}{ Table 3. Serum Level of Electrolytes and Hospital Stay } \\
\hline
\end{tabular}

Serum sodium level $<136$ with NIV required were 10 (27.7\%), serum sodium level with $136-148$ and NIV requirement were $3(21.4 \%)$ and serum potassium level $<3.6$ and NIV requirement were $9(30 \%)$ and 3.6-5 were 4 and serum magnesium $<108$ were $8(34.7 \%), 1.8-2.6$ were 4 (15.38\%) and serum magnesium level $>2.6$ were 1 (100\%).

Decreasing electrolyte levels demands Non-invasive ventilation (Table 4). At normal level of electrolytes (sodium3 , potassium-4, magnesium-4) few people required noninvasive ventilation. But when the electrolytes reduced below the lower limit, more number of patients required noninvasive ventilation (sodium $<136 \mathrm{mEq} / \mathrm{L}-10$ patients, potassium $<3.6 \mathrm{mEq} / \mathrm{L}-9$ patients, magnesium $<1.8 \mathrm{mEq} / \mathrm{L}$ 8 patients). This study shows that 49 IP patients required hospital stay more than 5 days (Table 4). More Days of Hospital stay is required for Serum Sodium level $<136-36$ 
Patients, Serum Potassium $<3.6$ - 30 Patients and Serum Magnesium level 1.8 to 2.6 - 25 Patients.

\begin{tabular}{|c|c|c|c|c|c|}
\hline \multicolumn{2}{|c|}{ Serum } & \multicolumn{2}{c|}{ NIV } & \multirow{2}{*}{ Total } & \multirow{2}{*}{ p-value } \\
\cline { 2 - 5 } & No & Yes & & \\
\hline \multirow{2}{*}{$\begin{array}{c}\text { S. Sodium } \\
\text { (mEq/L) }\end{array}$} & $<136$ & 26 & 10 & 36 & \multirow{2}{*}{0.646} \\
\cline { 2 - 5 } & $136-148$ & 11 & 3 & 14 & \\
\cline { 2 - 5 } & $>148$ & 0 & 0 & 0 & \\
\hline \multirow{2}{*}{$\begin{array}{c}\text { S. Potassium } \\
\text { (mEq/L) }\end{array}$} & $<3.6$ & 21 & 9 & 30 & \multirow{2}{*}{0.430} \\
\cline { 2 - 5 } & $3.6-5$ & 16 & 4 & 20 & \\
\cline { 2 - 5 } & $>5$ & 0 & 0 & 0 & \\
\hline \multirow{2}{*}{$\begin{array}{c}\text { S. Magnesium } \\
\text { (mEq/L) }\end{array}$} & $<1.8$ & 1 & 8 & 23 & \multirow{2}{*}{0.071} \\
\cline { 2 - 5 } & $1.8-2.6$ & 2 & 4 & 26 & \\
\cline { 2 - 5 } & $\geq 2.6$ & 0 & 1 & 1 & \\
\hline Table 4. Serum
\end{tabular}

This study shows that out of 50 inpatients, 16 (32\%) required readmission within 6 months. In inpatients, 11 patients with Serum Sodium level $<136$ (Hyponatraemia) required readmission. In inpatients, 8 Patients with Serum Magnesium level <1.8 (Hypomagnesaemia) required readmission. Among 50 inpatients, 23 patients were Hypomagnesaemic patients. Out of 23, 8 were readmitted showing lower magnesium levels in exacerbated COPD. Outcome shows, in 50 inpatients, 49 patients survived and one died. Although dyselectrolytaemia was more frequent in inpatients it was not statically significant.

\section{DISCUSSION}

In this study, in outpatients, $66 \%$ were smokers, $16 \%$ were past smokers and $18 \%$ were non-smokers. In inpatients, $68 \%$ were smokers, $12 \%$ were past smokers and $20 \%$ were nonsmokers. Many studies suggested that smoking is the commonest aetiological factor for COPD. [1,2,]

Hyponatraemia is typically observed in the final stages of chronic obstructive pulmonary disease (COPD). Patients with COPD are susceptible to hyponatraemia for a number of reasons. Chronic hypoxia and hypercapnia secondary to the underlying pulmonary illness, heart failure or renal insufficiency, use of diuretics, use of bronchodilators or steroids, malnutrition, and poor intake during acute exacerbations are common contributing factors for hyponatraemia in such patients. Activation of the reninangiotensin-aldosterone system and inappropriately elevated plasma arginine vasopressin (AVP) in COPD may cause hyponatraemia.[3]

Irrespective of the underlying mechanism of development, hyponatraemia itself may be a predictor of poor outcome in patients of COPD. It may lead to central nervous system dysfunction; confusion, convulsions, coma, reversible cardiac conduction defect, secondary renal insufficiency even death.[4,5] Therefore, hyponatraemia should be meticulously searched for in every patient with acute exacerbation of COPD and should be actively tackled at the earliest. In our study, out of 50 inpatients, 30 (72\%) were with hyponatraemia. Out of 50 outpatients, 14 (28\%) also had hyponatraemia. Along with hyponatraemia, hypokalaemia may be another morbid accompaniment in the subjects with COPD. Hypokalaemia may be present independently or concomitantly with hyponatraemia. In our study, there was a significantly low level of serum potassium in COPD in patients than the stable COPD. Hypokalaemia in
COPD may be attributed to respiratory acidosis and metabolic alkalosis or longstanding steroid therapy.[6] Use of beta 2adrenoceptor agonists like fenoterol or salbutamol may also contribute to hypokalaemia in COPD patients.[7] Moreover, acute respiratory failure associated with hypokalaemia was found to have a high mortality rate among the COPD patients. ${ }^{[8]}$ High mortality in hypokalaemia may be attributed to cardiac arrhythmias or hampered nerve-muscle conduction. So, it appears from our study that hypokalaemia may be a common associated finding in the subjects with COPD that should be corrected promptly to avoid fatal outcomes.

Since magnesium is involved in muscle tone, a decrease in magnesium level in COPD represents a factor which decreases respiratory muscle function and induces muscle fatigue. COPD represents an overlap of chronic bronchitis and emphysema and patients of COPD have an element of hyperresponsive airways. Misbalance of $\mathrm{Mg}$ serum concentration is not a rare appearance in patients with pulmonary diseases. Results from literature described frequency of hypomagnesaemia in $60 \%$ among hospital treated patients, especially in patients who were medically treated in Intensive Care Units. Greater mortality was seen in patients with hypomagnesaemia, compared with patients who had normal concentrations, especially when they were treated in Intensive Care Unit. In IP, AECOPD patients were with normal serum magnesium level and 23 had hypomagnesaemia. Serum magnesium is an independent predictor of frequent hospital readmissions. [9]

So there is a strong evidence from literature indicating low magnesium is an important cause for hospitalisation or it even interferes with prognosis. So our study strongly recommends immediate correction of electrolyte levels especially magnesium in the inpatient setting and daily monitoring of electrolyte levels in hospitalised COPD patients.

\section{CONCLUSION}

Chronic Obstructive Pulmonary Disease (COPD) is one of the leading causes of mortality and morbidity worldwide. Due to lack of awareness about the precipitating factors and predictors of prognosis, cases of acute exacerbation of COPD often suffer the fatal outcomes.

In this study, we assessed the levels of serum sodium and potassium in subjects with acute episodes of COPD and their stable controls. We found a significantly low level of serum sodium, potassium and magnesium in subjects with acute exacerbation of COPD than stable COPD patients.

As COPD is not fully reversible with medications, we need to look into the modifiable factors such as serum sodium, potassium and magnesium to reduce morbidity and mortality. Risk of readmission is higher in Acute Exacerbated Chronic Obstructive Pulmonary Disease patients with hyponatraemia, hypokalaemia and hypomagnesaemia.

Risk of the disease can be reduced by educating and empowering patients/families to manage COPD and comorbid conditions and this is essential to effective patient care management. Ensure appropriate treatment for COPD exacerbations while hospitalised. GOLD Guidelines recommend steroids, antibiotics for most patients, along with bronchodilators. Appropriate long-acting maintenance therapy should be prescribed prior to discharge. Educate patients on inhaler techniques, managing supplementary 
oxygen, drug regimens to improve respiratory status, pharmacologic (drug) and non-pharmacologic (e.g. pursed lip breathing) regimens and continuation of smoking cessation counselling. Reconcile medications and ensure that the patient has access to these medicines at the time of discharge. If spirometry has not been done to confirm the diagnosis of COPD, or has not been done recently, spirometry testing should be arranged shortly after the patient stabilises from the COPD exacerbation to help stratify the severity of their disease (GOLD Grade classification).

In this study, serum sodium, potassium and magnesium levels were deranged in subjects with acute exacerbations of COPD which should be routinely checked to avoid fatal outcomes. Hence, our study findings indicate that dyselectrolytaemia has an impact in the management of COPD patients.

\section{REFERENCES}

[1] Jindal SK, Agarwal AN, Gupta D. A review of population studies from India to estimate national burden of chronic obstructive pulmonary disease and its association with smoking. Indian J Chest Dis Allied Sci 2001;43(3):139-47.

[2] Jindal SK, Aggarwal AN, Chaudhry K, et al. A multicentric study on epidemiology of chronic obstructive pulmonary disease and its relationship with tobacco smoking and environmental tobacco smoke exposure. Indian J Chest Dis Allied Sci 2006;48(1):23-9.
[3] Bauer FK, Telfer N, Herbst HH, et al. Hyponatremia and increased exchangeable sodium in chronic obstructive lung disease. Am J Med Sci 1965;250(3): 245-53.

[4] Suri P, Habeeb K, Alai MS, et al. Hyponatremia Presenting as Cardiac Conduction Defect. J K Science 2009;11(2):85-6.

[5] Porcel A, Díaz F, Rendón P, et al. Dilutional hyponatremia in patients with cirrhosis and ascites Arch Intern Med 2002;162(3):323-8.

[6] Saini V, Saini N, Kaur J, et al. Acid-base status in chronic obstructive pulmonary disease patients. Ind J Clin Biochem 2008;28:36-8.

[7] Yang CT, Lin HC, Lin MC, et al. Effect of beta 2 adrenoceptor agonists on plasma potassium and cardiopulmonary responses on exercise in patients with chronic obstructive pulmonary disease. Eur J Clin Pharmacol 1996;49(5):341-5.

[8] Hussain SF, Irfan M, Naqi YS, et al. Acute respiratory failure in Pakistani patients: risk factors associated with mortality. J Coll Physicians Surg Pak 2006;16(4):287-90.

[9] Bhatt SP, Khandelwal P, Nanda S, et al. Serum magnesium is an independent predictor of frequent readmissions due to acute exacerbation of chronic obstructive pulmonary disease. Respiratory Medicine 2008;102(7):999-1003. 